\title{
Quantum Interference and Confinement Phenomena in Mesoscopic Superconducting Systems
}

\author{
V. V. Moshchalkov, L. Gielen, M. Baert, V. Metlushko, G. Neuttiens, C. Strunk, V. Bruyndoncx, X. Qiu, M. Dhallé, \\ K. Temst, C. Potter, R. Jonckheere, ${ }^{*}$ L. Stockman, M. Van Bael, C. Van Haesendonck and Y. Bruynseraede
}

Laboratorium voor Vaste-Stoffysika en Magnetisme, Katholieke Universiteit Leuven, Celestijnenlaan 200 D, B-3001 Leuven, Belgium

Received April 25, 1994; accepted May 11, 1994

\begin{abstract}
The superconducting field $(H)$-temperature $(T)$ phase boundary has been measured in mesoscopic $\mathrm{Al}$ samples of different topology: lines, open and filled squares, which were made under the same conditions from the same material. These samples clearly show different superconducting $H-T$ phase boundaries which are nicely reproducing the predictions of the theoretical calculations made for their particular confinement geometries. The confinement of the flux lines by the lattice of the submicrometer holes has been studied in the $\mathrm{Pb} / \mathrm{Ge}$ multilayers. A substantial enhancement of the critical current $j_{c}$ has been achieved. Sharp integer and rational matching peaks in the $j_{c}(H)$ curve are observed. The possibility of the "quantum design" of the superconducting critical parameters $\left(H_{c}(T)\right.$ and $\left.j_{c}(T, H)\right)$ of the mesoscopic and nanostructured superconductors by optimizing the confinement geometry for the superconducting condensate and for the flux lines has been demonstrated.
\end{abstract}

\section{Introduction}

The superconducting state can be suppressed by applying magnetic field $H$ or increasing temperature $T$. It is generally believed that the $H-T$ line separating the superconducting from the normal state is mainly determined by the material itself. This is true for bulk samples, where the surface to volume ratio is quite small and the effect of the boundary conditions on the formation of the superconducting state can be neglected $[1,2]$. In mesoscopic superconducting samples, however, the surface to volume ratio is so large that the nucleation of the superconducting state is strongly dependent upon the boundary conditions imposed by the specific sample shape. The confinement geometry for the superconducting condensate plays a role similar to that of the confinement potential in the quantum mechanical problem "particle in a box". As a result, the superconducting $H-T$ phase boundary can be intentionally designed by choosing the proper sample topology, like the eigenvalues of a quantum particle can be tuned by changing the confinement potential.

In the first part of this paper we have checked this idea experimentally by measuring the superconducting $H-T$ phase boundary in mesoscopic Al samples of different topology: lines, open and filled squares. These samples, all made from the same material under the same conditions and having the same $T_{c}$ values, clearly demonstrate different superconducting $H-T$ phase boundaries which nicely reproduce the theoretical calculations made for their proper confinement geometries. This proves that the topology of the

* Present address: Interuniversity Microelectronics Center, Kapeldreef 75, B-3001 Leuven, Belgium. mesoscopic superconducting samples strongly effects their critical parameters. We limit ourselves in this paper only with the orbital pairbreaking effects and will not consider the spin pairbreaking which will play a dominant role at $T \rightarrow 0$.

In the second part of this paper we focus on the flux lines confinement by the lattice of the artificially fabricated submicrometer holes. The optimization of the confinement geometry leads to the strong enhancement of the pinning force and the critical current $j_{c}$. Besides the $j_{c}$ enhancement we have observed sharp matching effects resulting from the commensurability of the flux lattice with the available periodicity of the submicrometer holes lattice. Therefore by designing the proper confinement geometry for the superconducting condensate and for the flux lines both the critical fields $H_{\mathrm{c}}(T)$ and the critical currents $j_{\mathrm{c}}(T, H)$ can drastically be improved, i.e. "quantum design" of the superconducting critical parameters is possible.

\section{Confinement effects in mesoscopic superconducting lines, loops and dots}

The linearized Ginzburg-Landau equation, used to describe the nucleation of the superconducting state in the presence of a magnetic field $\boldsymbol{H}=\operatorname{rot} \boldsymbol{A}[1,2]$ :

$\frac{1}{2 m}\left(-\mathrm{i} \hbar \nabla-\frac{e^{*}}{c} A\right)^{2} \psi_{s}=-\alpha \psi_{s}$

is very similar to the Schrödinger equation for a particle with charge $e$ :

$\frac{1}{2 m}\left(-\mathrm{i} \hbar \nabla-\frac{e}{c} A\right)^{2} \psi=E \psi$

The superconducting analogue $\psi_{s}$ of the wave function $\psi$ is called the order parameter and its modulus is proportional to the density $n_{s}$ of the Cooper pairs $\left(\left|\psi_{s}\right|^{2}=\psi_{s} \psi_{s}^{*} \propto n_{S}\right)$ having the charge $e^{*}=2 e$ (eq. 1). The parameter $-\alpha$ in eq. (1) $[1,2]$ :

$-\alpha=\frac{\hbar}{2 m \xi^{2}(T)}=\frac{\hbar^{2}}{2 m \xi^{2}(0)} \frac{T_{c}-T}{T_{c}}$,

plays the same role for the order parameter $\psi_{s}$ (eq. (1)) as the eigenvalue $E$ for the wave function $\psi$ (eq. (2)). Here in eq. (3) $\xi(T)$ is the temperature dependent coherence length. The lowest $E$ values gives the highest possible temperature for the onset of the superconducting state. Therefore, the superconducting $H-T$ phase boundary can be found by: (i) solving the corresponding Schrödinger equation, (ii) taking 
the lowest $E(H)$ state, (iii) rotating $E(H)$ scheme by $90^{\circ}$ to obtain finally the superconducting $H(T)$ phase line.

For bulk superconducting samples, where the effect of the sample boundaries upon the nucleation of the superconducting state can be neglected, this scheme defines the upper critical field $H_{c 2}(T)$ (Fig. 1). In this particular case the solution of the Schrödinger equation gives the Landau levels with the lowest state $E(H)=\hbar \omega / 2$ [Fig. 1(a)]. Rotation of this $E v s$. $H$ plot by $90^{\circ}$ gives the $H_{c 2}(T)$ line [see Fig. 1(b)].

In mesoscopic superconducting samples the surface to volume ratio is quite large and the boundary conditions become very important. The boundaries of the superconducting samples determine the confinement geometry for the superconducting condensate. For the superconductorvacuum interface the order parameter $\psi_{s}$ obeys the following boundary conditions for the normal component of the superfluid velocity $v_{S}=-\mathrm{i} \hbar \nabla-\left(e^{*} / c\right) A[1,2]$ :

$\left.\left(-\mathrm{i} \hbar \nabla-\frac{e^{*}}{c} A\right)\right|_{n} \psi_{s}=0$,

i.e. the supercurrent can not flow perpendicular to the sample boundary. Therefore it is evident that by changing the actual sample topology which enters eq. (4), we can strongly modify the lowest energy level $E(H)$ and also the superconducting $H-T$ line. This unique possibility, however, can only be realized in mesoscopic superconducting systems with the characteristic size $L$ being smaller than both the coherence length $\xi(T)$ and the penetration depth $\lambda(T)$. In bulk superconductors with $L \gg(\xi(T), \lambda(T))$ the modification of the sample shape will only influence the surface superconducting layer, which appears at the third (surface) critical field $H_{c 3}(T)$ [1-3], and will not change the bulk superconducting state below the upper critical field $H_{c 2}(T)$.

In order to check the effect of the topology of the mesoscopic samples upon these superconducting critical parameters we prepared filled squares with a side of $1 \mu \mathrm{m}$, lines with a width $w=0.15 \mu \mathrm{m}$ and open squares with a side of $1 \mu \mathrm{m}$ and width $w=0.15 \mu$, (Fig. 2). These mesoscopic structures were made with a left-off technique. All the fabricated Al samples have a film thickness $t=0.025 \mu \mathrm{m}$. The Al films were obtained by thermal evaporation of high purity $\mathrm{Al}(99.9995 \%)$ in a reduced helium atmosphere ( $p=10^{-3}$ Torr), which, according to our experience, produces smooth and homogeneous metallic films. The electrical transport measurements are performed in a temperature stabilized helium -4 cryostat. The square structures typically

(a)

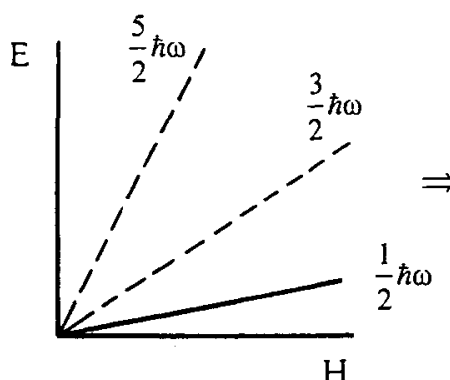

(b)

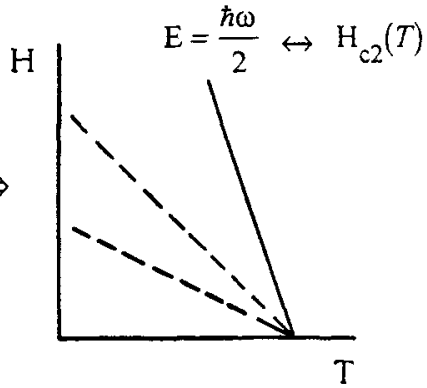

Fig. 1. (a) Energy vs. field for bulk samples. The solid line corresponds to the lowest energy value. (b) The lowest Landau level defines the upper critical field $H_{c 2}(T)$ which is obtained by rotating the $E(H)$ plot by $90^{\circ}$.

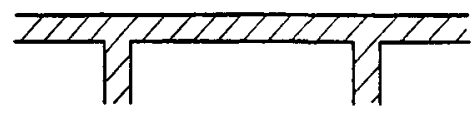

LINE

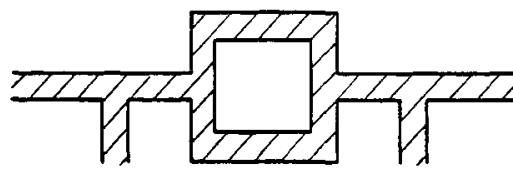

LOOP

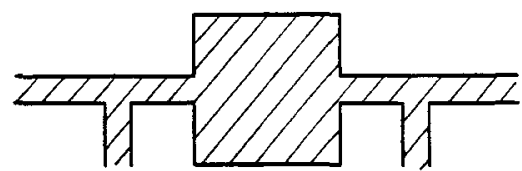

DOT

Fig. 2. The geometry of the investigated mesoscopic structures.

have a normal state resistance $R_{n} \cong 20 \Omega$ and for the line structure this parameter has the value $R_{n} \cong 10 \Omega$. The sample resistances were measured using a commercial fourterminal AC resistance bridge (Linear Research 400). The experimental procedure is described in more detail in Ref. [4].

Major cracks occurring at the grain boundaries in the $\mathrm{Al}$ lines, or discontinuities caused by a local failure of the liftoff process may induce a weak Josephson coupling between different sample areas. We are confident that with our preparation method the accidental formation of such weak links in the $\mathrm{Al}$ structures is very unlikely. Indeed, the resistivity $\rho \cong 4.2 \times 10^{-6} \Omega \mathrm{cm}$ of the Al structures as well as the resistance ratio $R(300 \mathrm{~K}) / R(4.2 \mathrm{~K}) \cong 2.1$ are comparable to the values measured for the much larger $\mathrm{Al}$ contact pads and indicate a pronounced metallic character. Moreover, detailed atomic force microscopy and scanning electron microscopy studies of the Al films confirm the presence of a smooth and continuous film surface.

The superconducting $H-T$ phase boundaries are reconstructed by measuring the temperature shift of the middle point of the resistive transition for different magnetic fields. The $H-T$ phase boundaries measured for Al samples with different topology, are shown in Figs 3 and 4. The magnetic field was always applied perpendicular to the structures. Figure 3 demonstrates the square root behaviour, $H_{c \|}(T) \propto$ $\sqrt{\left(1-\left(T / T_{c}\right)\right)}$, which was expected for the superconducting line structure with a width $w$ smaller than the temperature dependent coherence length $\xi(T)=\xi(0) / \sqrt{\left(1-\left(T / T_{c}\right)\right)}$. The phase boundary of such a line structure with $w<\xi(T)$ in a perpendicular field, should be similar to that of a thin film in a parallel field, since in both cases the area exposed to the magnetic field has the same characteristic geometry. It is well known that for a plane in a parallel field the phase diagram behaves as [5],

$H_{c \|}(T)=\frac{\sqrt{12}}{2 \pi} \frac{\phi_{0}}{\mu_{0} w_{\text {eff }} \xi(0)} \sqrt{\left(1-\left(T / T_{c}\right)\right)}$,

where $T_{c}$ is the Ginzburg-Landau critical temperature.

The solid line in Fig. 3, which is in good agreement with our experimental data, represents the theoretical fit with the Tinkham formula for $\xi(0)=0.26 \mu \mathrm{m}$ and the effective width $w_{\text {eff }}=0.20 \mu \mathrm{m}$ close to the real width of the line. The value for $\xi(0)$ found from the dirty limit expression $\xi(0)=$ 


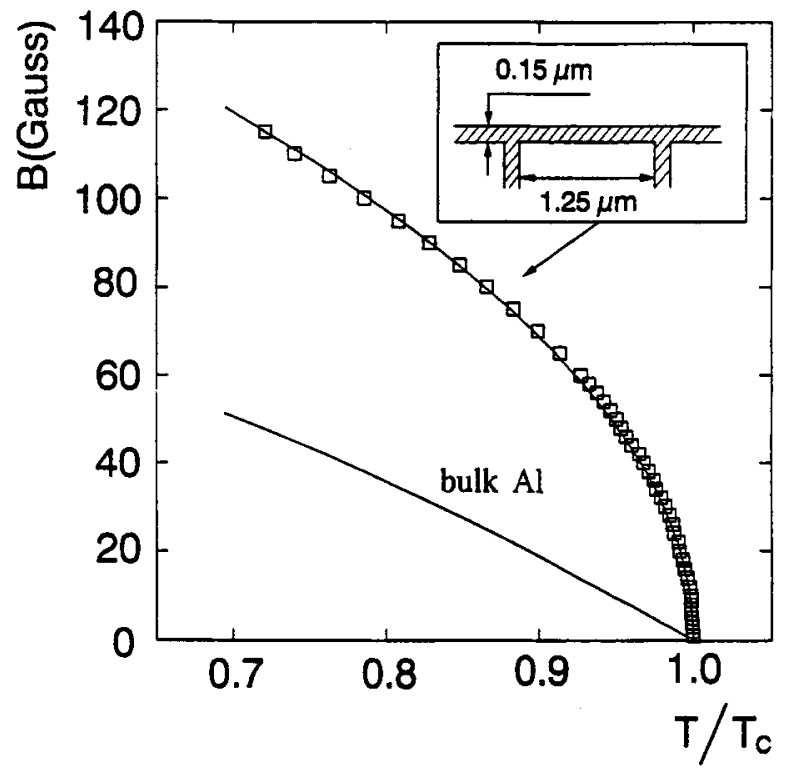

Fig. 3. The measured superconducting phase boundary $T_{c}(H)$ as a function of the reduced temperature $T / T_{c}$, for the line with length $l=1.25 \mu \mathrm{m}$ and a width $w=0.15 \mu \mathrm{m}$. The solid line is calculated by using the relation for the upper critical field $H_{c||}(T)$ for a thin film (eq. (5)) with $w_{\text {eff }}=0.26 \mu \mathrm{m}$. The dashed line represents the phase boundary for bulk $\mathrm{Al}\left(H_{\mathrm{c}}(T \rightarrow 0)=\right.$ $100 \mathrm{Oe}$ ). The inset shows the geometry of the investigated $\mathrm{Al}$ line.

$0.85\left(\xi_{0} l\right)^{1 / 2} \cong 0.20 \mu \mathrm{m}$ and $l \cong 22 \mathrm{~nm}$ estimated from the normal state resistivity $\rho \cong 4.2 \times 10^{-6} \Omega \mathrm{cm}$ is also in agreement with the coherence length $\zeta(0)=0.26 \mu \mathrm{m}$ derived from the coefficient describing the square root curve.

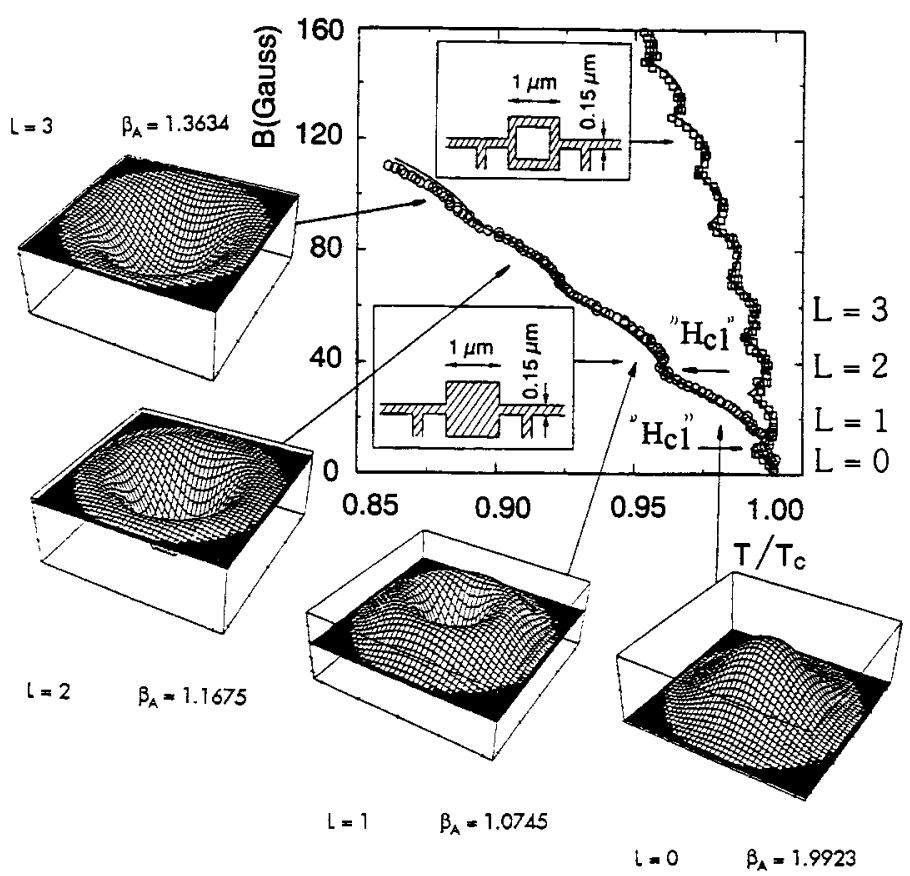

Fig. 4. The measured superconducting phase boundary $T_{c}(H)$ as a function of the reduced temperature $T / T_{c}$, for the $1 \times 1 \mu \mathrm{m}^{2}$ open Al square ( $\square$ ) and for the $1 \times 1 \mu \mathrm{m}^{2}$ filled $\mathrm{Al}$ square $(O)$. The solid lines correspond to calculations based on Tinkham formula for an open square and a disk by using eq. (1) with the boundary conditions given by eq. (4) [5]. The insets show the geometries of the open and filled $\mathrm{Al}$ squares. The change of the amplitude of the order parameter $|\psi|^{2}$ for different values of the orbital quantum number $L$ are represented in three-dimensional plots. As $L$ increases, multiquanta vortices are formed in the center of the sample. The Abrikosov parameter $\beta_{\mathrm{A}}$ [2] is also given for various patterns of the order parameter.
The superconducting loop (Fig. 4) demonstrates the classical Little-Parks oscillatory $H-T$ phase boundary [6], related to the quantization of the flux threading the loop area. Again, the theoretical phase boundary, found by considering the actual sample geometry, describes very well the experimental data (see solid line in Fig. 4). Moreover, in this simple homogeneous superconducting loop the two supercurrents $I_{1}$ and $I_{2}$ flowing in the different branches of the loop again opposite phases $\mp \pi \phi / \phi_{0}$ from the perpendicular applied field [7].

$I_{1} \propto \sin \left(\beta-\alpha-\pi \frac{\phi}{\phi_{0}}\right)$

$I_{2} \propto \sin \left(\beta-\alpha+\pi \frac{\phi}{\phi_{0}}\right)$.

The interference of the currents flowing through the two branches of the loop produces an oscillatory dependence of the total current $I=I_{1}+I_{2}$;

$I \propto \sin (\beta-\alpha) \cos \left(\pi \frac{\phi}{\phi_{0}}\right)$.

The current amplitude in eq. (8) is determined by the phase difference $\Delta \varphi=\beta-\alpha$ (as in the first Josephson equation $I \propto \sin \Delta \varphi)$ [1, 2]. The critical current $I$ for the homogeneous loop is obtained by taking $\sin \Delta \varphi=1$ in eq. (8) [7]:

$I_{c} \propto \cos \left(\pi \frac{\phi}{\phi_{0}}\right)$.

Equation 9 implies that the critical current of a mesoscopic superconducting loop without artificial Josephson weak links oscillates with the applied magnetic field in the same way as it does in a classical SQUID with extrinsic weak links. The existence of these oscillations has been confirmed experimentally in Ref. 8.

Now we are coming to the description of the quantization effects in the superconducting filled square (Fig. 4). Note that the external size of this square and the thickness are exactly the same as the dimensions of the open square. Evidently the $H-T$ boundary for the filled square differs much from the $H-T$ boundary for the open square. First of all, the field suppression of the superconducting state is much stronger in the filled square, which can easily be explained by the difference in the sample areas which are penetrated by the magnetic field. Secondly, the period $\Delta H$ of the oscillations superimposed with the nearly linear $T_{c} v s$. $H$ curve is noticeably larger than the field $\Delta H=20.2 \mathrm{Oe}$ calculated just from the total area $S$ of the filled square $\Delta H=\phi_{0} / S$.

It is clearly seen that the first period $H_{1} \cong 36.4 \mathrm{Oe}$ is larger than the others. The main experimental observations for the filled square can be summarized as follows: (i) nearly linear $T_{c}(H)$ boundary, superimposed with the $T_{c}(H)$ oscillations, (ii) the period of the $T_{c}(H)$ oscillations is different from that of an open square with the same external dimensions, (iii) the first period is larger.

All these observations are fully supported by the theoretical calculations using eq. (1) with proper boundary conditions (eq. (4)). The energy levels $E v s$. field $H$ for a disk (which we consider here as an analogue of the filled square) are shown in Fig. 5. The detailed description of $E(H)$ can be found in Ref. [3]. These levels correspond to different orbital quantum numbers $L$ in the solution for the order 


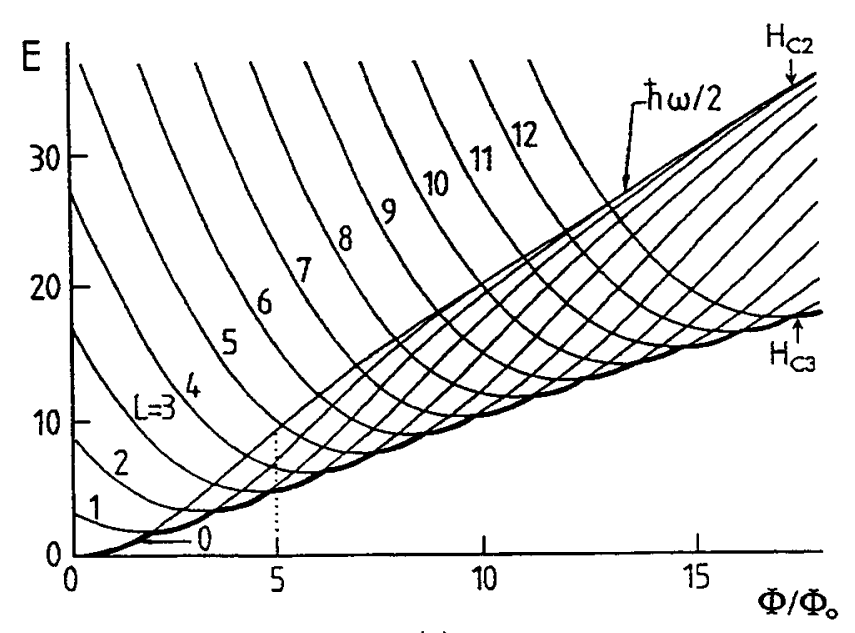

(a)

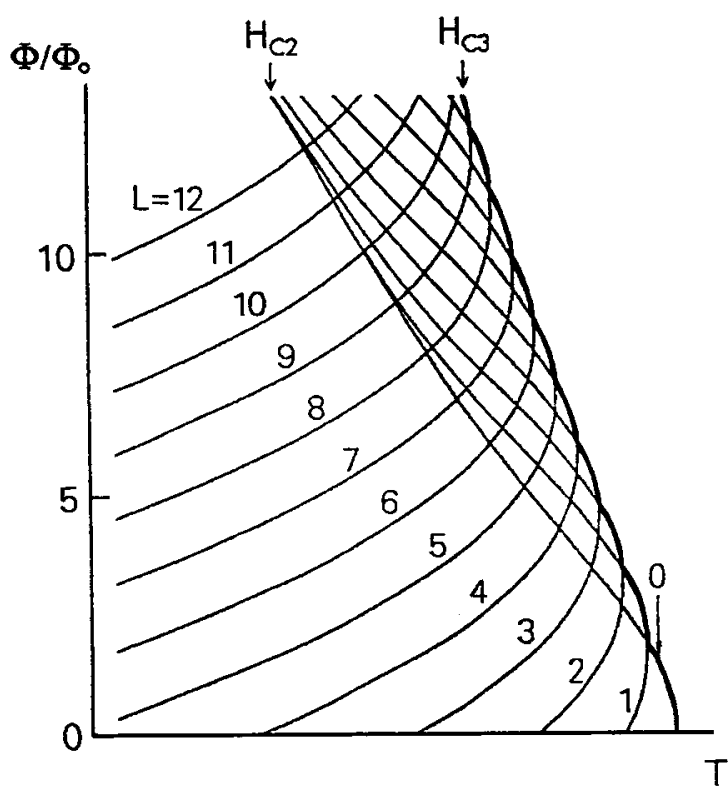

(b)

Fig. 5. Solutions of the Ginzburg-Landau eq. (1) for the disk with the boundary conditions (4) for the angular momenta $L$ as function of the reduced applied magnetic flux $\phi / \phi_{0}$. (a) The energy levels as function of $\phi / \phi_{0}$ for a disk. (b) The thick solid line, in the rotated scheme, corresponds to the superconducting $T_{c}(H)$ phase boundary. The oscillations are due to the change of the orbital quantum number $L$.

parameter $\psi$ taken in the form of a product of the radial $f(r)$ and the angular $\left(\mathrm{e}^{-i L \varphi}\right)$ dependence: $\psi_{s}=f(r) \mathrm{e}^{-i L \varphi}$. The solid line [Fig. 5(a)], giving us the lowest energy, thus corresponds to the superconducting $T_{c}(H)$ phase boundary [Fig. 5 (b)] with oscillations arising from the change of the orbital momentum $L$ [3]. The calculations reproduce remarkably well both nearly linear $H-T$ suppression (see the solid line in Figs 4 and 5b) and the oscillations, including unusual difference between the first period and the others (see also Table I). It is worth noting here that due to the confinement effect the energy levels for a dot differ very much from the

Table I. Comparison between the experimental and theoretical values of the periodicity of the reduced magnetic $\phi / \phi_{0}$ flux for the filled square

\begin{tabular}{llllll}
\hline Period & 1 & 2 & 3 & 4 & 5 \\
\hline$\phi / \phi_{0}$ (exp.) & 1.8 & 3.3 & 4.7 & 5.9 & 7.2 \\
$\phi / \phi_{0}$ (theory) & 1.9 & 3.4 & 4.7 & 6.0 & 7.3 \\
\hline
\end{tabular}

Landau levels found for infinite samples [compare Fig. 1(a) and 5(a)].

The analogue of the lower critical field $H_{c 1}$ for mesoscopic structures (Fig. 4) can be found from the transition $L=0 \rightarrow L=1$. We clearly see again the qualitative difference between a bulk and a mesoscopic superconductor: in the former the $H_{c 1}$ value is determined by the material properties, whereas in the latter $H_{c 1}$ is found from the fluxoid quantization and is strongly dependent upon the sample geometry (compare " $H_{c 1}$ " for an open and a filled square, Fig. 4).

By making a direct comparison of the superconducting samples of different topology fabricated from the same superconducting material under the same conditions (lines, open and filled squares, with the same $T_{c}$ ), we may conclude that these $T_{c}(H)$ phase boundaries are fully governed by confinement and quantization effects. This implies that the actual sample geometry imposes well defined boundary conditions (eq. (4)) for the solution of the analogue of the Schrödinger equation for the superconducting order parameter $\psi_{s}$ (eq. (1)). The modification of the confinement geometry drastically influences the quantization and therefore the $T_{\mathrm{c}}(H)$ boundary. As a result, the slope and the shape of the $T_{c}(H)$ boundary can be changed considerably just by taking proper sample geometry. For example the $T_{c} v s$. $H$ slope may be maximized by minimizing the area exposed to the magnetic field. All these observations convincingly demonstrate the practical possibilities of controlling the superconducting critical parameters by optimizing the topology of the samples of the mesoscopic length scale; i.e. "quantum design" of the $T_{c}(H)$ line is possible.

\section{Confining the flux lines by the lattice of submicrometer holes}

The geometries and the basic equations for the two problems: lattice of superconducting dots (Fig. 6) and lattice of holes in a thin superconducting film (Fig. 7) are very similar. For example the orbital quantum numbers $L$ used to describe the quantization effects in the superconducting dot (Fig. 4) correspond to multi-quanta vortices trapped by the holes. Confinement of the flux lines by the lattice of artifically fabricated submicrometer holes can result in a substantial enhancement of $j_{c}$. Indeed, the theoretical upper limit of

\section{Lattice of dots}

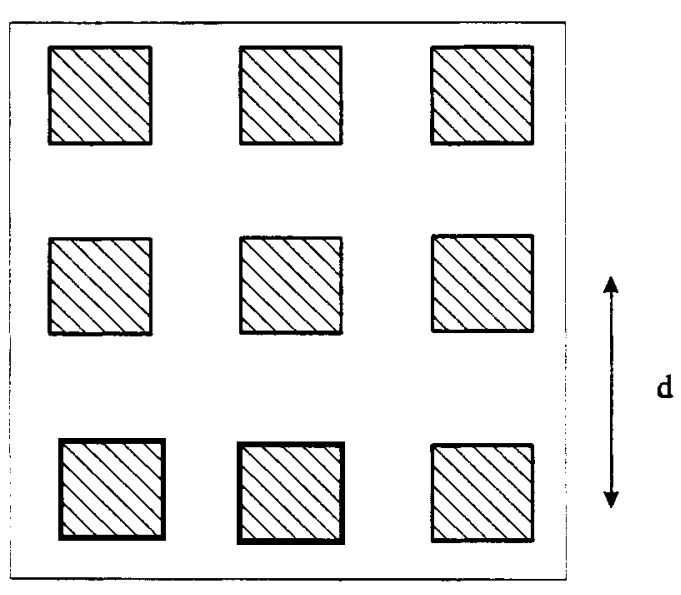

Fig. 6. A square lattice of dots with the period $d$. 


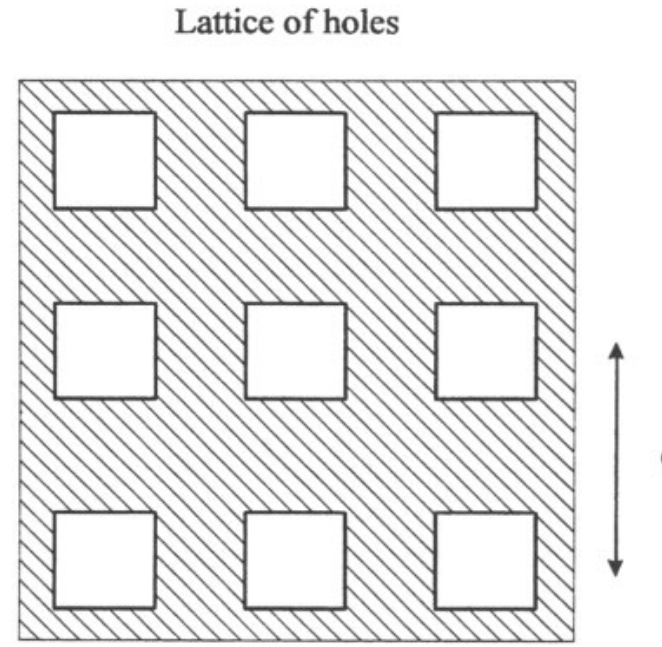

Fig. 7. A quare lattice of holes with period $d$.

the critical current density $j_{c}$ in superconductors is determined by the depairing current $j_{0}=c \Phi_{0}\left(12 \pi^{2} \sqrt{3} \lambda^{2} \xi\right)^{-1}$, where $\Phi_{0}$ is the flux quantum, $\xi$ the coherence length and $\lambda$ the penetration depth [2]. The experimental $j_{c}$ value, however, can be orders of magnitude lower since $j_{c}$ is not only related to $\lambda$ and $\xi$ but also to the strength of the pinning force $f_{p}$. In a superconductor without pinning centers, $f_{p} \sim 0$ and $j_{c}$ is extremely low whereas in a superconductor with a high density of proper pinning centers the $j_{c}$ value can approach its theoretical limit $j_{0}$. This implies that by optimizing the density and the characteristics of the pinning centers, $j_{c}$ may be enhanced by orders of magnitude [9]. Different types of artificial pinning centers have been proposed such as a random distribution of point defects [10] and columnar amorphous tracks $[11,12]$, or regular thickness modulations [13].

The main idea of the optimisation scheme is the creation of defects with a size comparable to the vortex core $\xi$ and with a density of the order of the number of flux lines per unit area $n_{v} \approx H / \Phi_{0}$. A possibile way to create efficient pinning centers and to enhance $j_{c}$ substantially is to fabricate a lattice of very small holes in the superconductor $[14$, 15]. Simultaneously this two-dimensional pattern of holes can be used to investigate resonant pinning and also possible rational matching peaks. Matching effects appear as a result of a commensurability between a periodic defect structure and a flux line lattice. At first sight a lattice of holes can be simply considered as a limiting case of a superconducting network, with an increased thickness of wires or a decreased empty space area between the wires. However, contrary to wire networks [16-21], lattices of holes give a unique possibility to study (i) the repulsive interaction between the flux lines in presence of the periodic lattice of pinning centers and (ii) the dependence of the pinning potential at holes upon the number of flux lines trapped in each hole. In spite of these new possibilities, lattices of holes were seldom investigated $[14,15]$.

The temperature $(T)$ and field $(H)$ dependence of $j_{c}$ was only derived so far from transport measurements in single layers with holes, while SQUID magnetization measurements have not been used for this purpose. The study of the $j_{c}$ enhancement and matching effects in superconductors, especially in multilayers, with a well defined lattice of holes

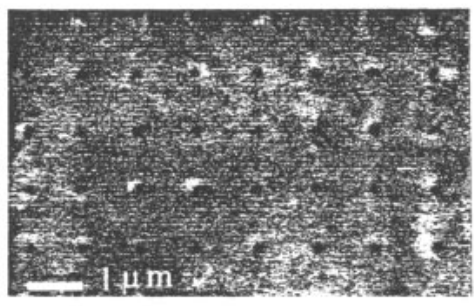

Fig. 8. SEM picture of the $[\mathrm{Pb}(150 \AA) / \mathrm{Ge}(140 \AA)]_{3}$ multilayer with a square lattice of holes with radius $0.1 \mu \mathrm{m}$ and the period $d=1 \mu \mathrm{m}$.

is very promising since one may expect a coherent accumulation of $j_{c}(H)$ of the matching peaks from each individual superconducting layer.

We have carried out detailed magnetization studies of the resonant enhancement of the pinning force and matching effects in $\mathrm{Pb} / \mathrm{Ge}$ multilayers, in which a square lattice of submicrometer holes is made (Figs 8 and 9). These holes act as efficient pinning centers $[14,15,22]$, trapping the vortices and enhancing the critical current density substantially.

$\mathrm{The} \mathrm{Pb} / \mathrm{Ge}$ multilayers are prepared by electron beam evaporation in a MBE apparatus with a base pressure of $2 \times 10^{-9}$ Torr [23]. Typical evaporation rates, controlled by a quadrupole mass spectrometer, are $5 \AA / \mathrm{s}$ for $\mathrm{Pb}$ and $1 \AA / \mathrm{s}$ for $\mathrm{Ge}$. In order to obtain continuous thin $\mathrm{Pb}$ films, the $\mathrm{SiO}_{2}$ substrates were kept at liquid nitrogen temperature during the evaporation. This low substrate temperature leads to the formation of texture $\mathrm{Pb}$ (111) layers and amorphous Ge layers. Layer thicknesses were monitored with quartz crystal oscillators during the evaporation and calibrated with a Dektak surface profilometer. An independent cross-check of the thickness calibration was obtained from $\mathrm{X}$-ray diffraction spectra. Simulations of these spectra show that the structure is well layered, with negligible interdiffusion. An Atomic Force Microscopy (AFM) study was also performed on single $\mathrm{Pb}$ films, which indicates a roughness of less than $20 \AA$. All $\mathrm{Pb} / \mathrm{Ge}$ samples have a $[\mathrm{Pb}(150 \AA) / \mathrm{Ge}(140 \AA)]_{3} \mathrm{Ge}$ structure, where 3 denotes the number of bilayers and the top film is always a $140 \AA$ protective $\mathrm{Ge}$ layer. The superconducting transition temperature of the multilayers is $T_{c}=7.1 \mathrm{~K}$. The square lattice of holes in the films is obtained by a lift-off technique using electron beam lithography. The distance between the holes

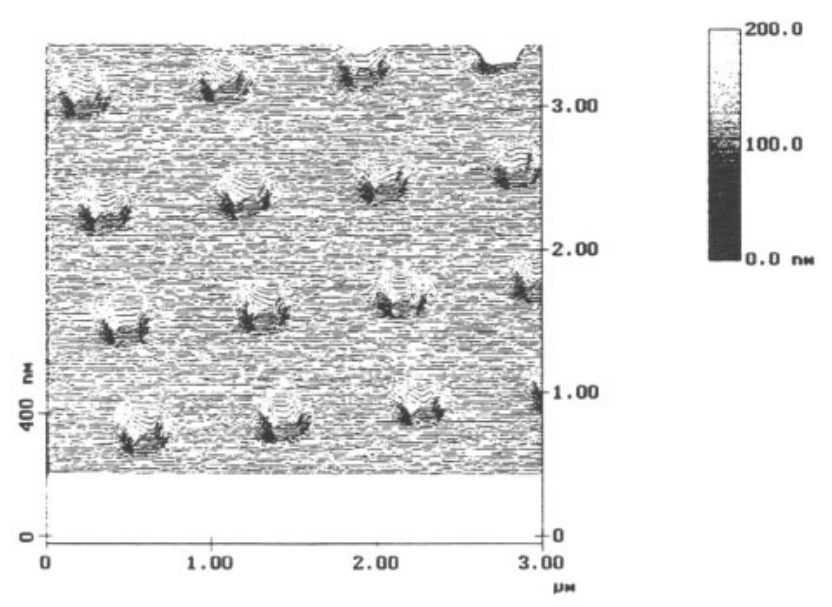

Fig. 9. AFM micrograph of the $[\mathrm{Pb}(150 \AA) / \mathrm{Ge}(140 \AA)]_{3}$ multilayer with a square lattice of holes with radius $0.2 \mu \mathrm{m}$ and the period $d=1 \mu \mathrm{m}$. 
is $d \approx 1 \mu \mathrm{m}$, the radius of the holes $r \approx 0.1 \mu \mathrm{m}$ (see SEM picture in Fig. 8) and $r \approx 0.2 \mu \mathrm{m}$ (see AFM micrograph in Fig. 9). For this particular configuration the matching fields are $H_{n}=n \Phi_{0} / d^{2}=n \times 20.7 \mathrm{G}$, where $n$ is integer number.

Magnetization measurements were performed in a commercial Quantum Design SQUID-magnetometer with a scan length of $3 \mathrm{~cm}$, corresponding to a field homogeneity better than $0.05 \%$. Fig. 10 shows the magnetization loops measured at different temperatures and in a magnetic field $H_{\perp}$ perpendicular to a $\mathrm{Pb} / \mathrm{Ge}$ multilayer with and without the lattice of holes. A pronounced difference in $M\left(H_{\perp}\right)$ behaviour between the two systems can be observed: (i) the amplitude of the irreversible part of $M$ proportional to $j_{c}$ is substantially enhanced by the presence of holes; (ii) very sharp peaks show up at the matching fields $H_{1}, H_{2}$ and $H_{3}$ at temperatures close to $T_{c}$; (iii) at lower temperatures the
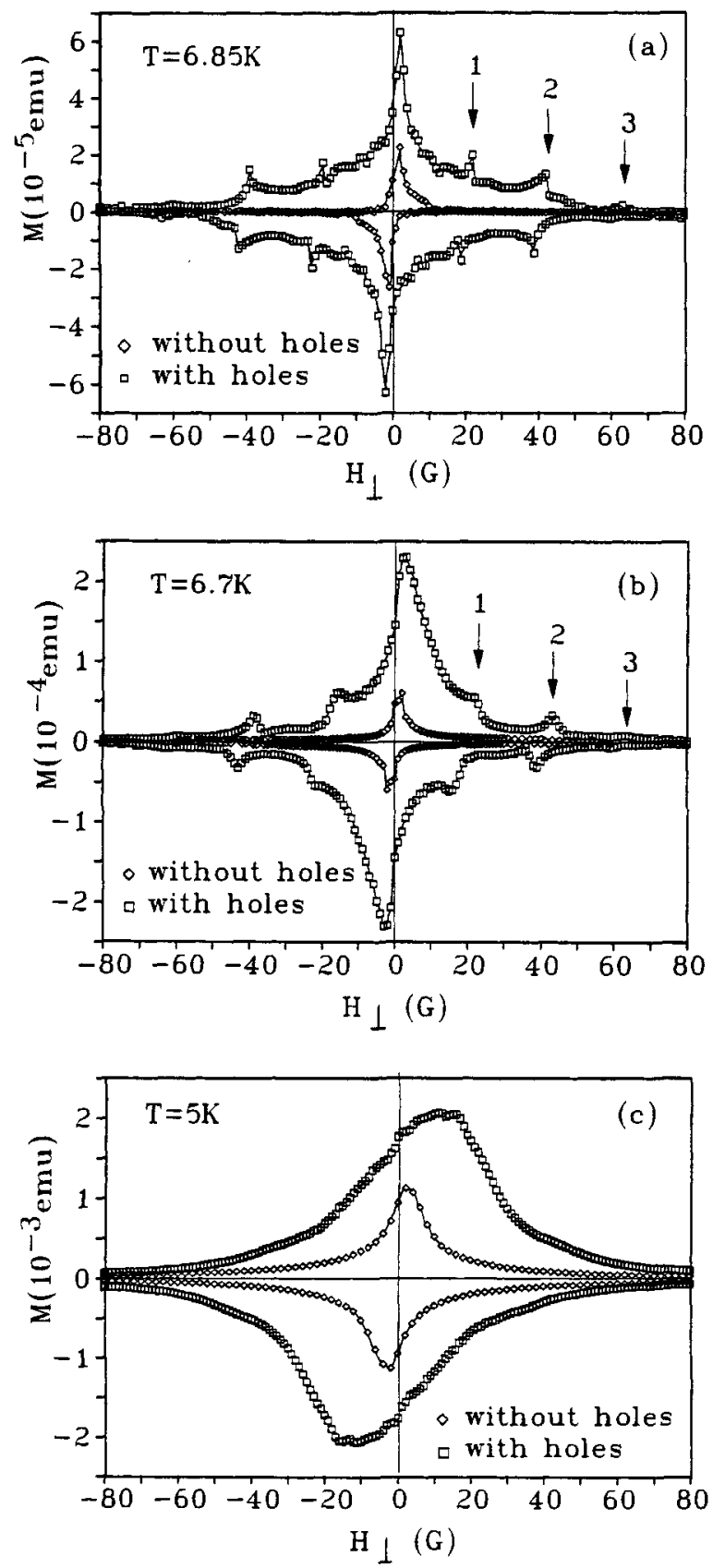

Fig. 10. Magnetization loops of a $[\mathrm{Pb}(150 \AA) / \mathrm{Ge}(140 \AA)]_{3}$ multilayer measured at different temperatures. The matching fields of the flux lines are indicated by the arrows. The data in Fig. 10(a)-(c) are obtained for the lattice of holes with $r=0.1 \mu \mathrm{m}$.

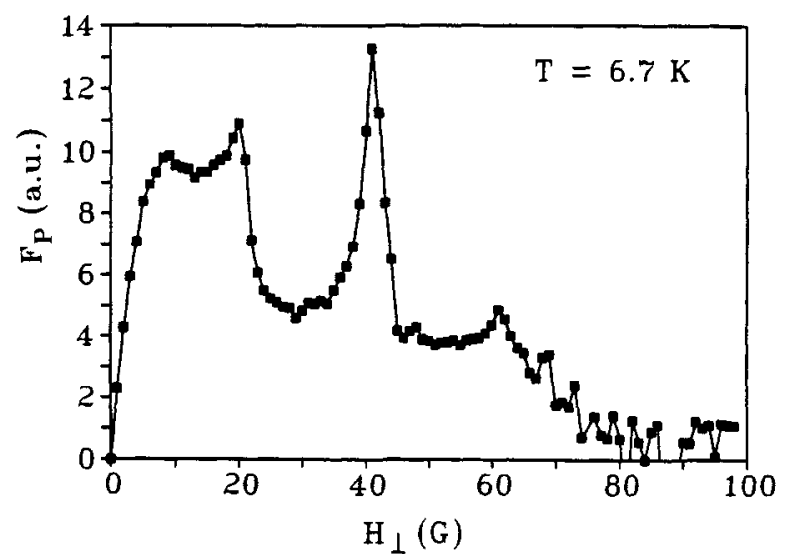

Fig. 11. The pinning force $F_{p} \approx j_{c} H$ of a $[\mathrm{Pb}(150 \AA) / \mathrm{Ge}(140 \AA)]_{3}$ multilayer with a square lattice of $0.1 \mu \mathrm{m}$ holes at $T=6.7 \mathrm{~K}$.

amplitude of the matching peaks decreases and they finally disappear at $T<6 \mathrm{~K}$ [Fig. 10(c)]. The pinning force $f_{p}$ also demonstrates sharp resonances at matching fields (see Fig. 11).

The field dependence of $M$ at $T=6.85 \mathrm{~K}$ [Fig. 10(a)] shows, besides the integer matching peaks, also rational peaks at lower field values. The fine structure in the first period of $M\left(H_{\perp}\right)$ at $H / H_{1}=1 / 2 ; 1 / 4 ; 1 / 8(1 / 9$ ?); $1 / 16$ can be better resolved if we plot $\mathrm{d} M / \mathrm{d} H_{\perp}$ as a function of $H_{\perp}$ (see Fig. 12). The rational matching peaks are, within the experimental sensitivity, only observed in the first period $(H<$ $H_{1}$ ). This is in sharp contrast with the behavior of superconducting wire networks, where rational peaks are observed over many periods [16].

An analysis of these remarkable features is closely related to the flux pinning by holes which is strongly dependent upon the number of the flux lines $n_{\mathrm{V}}$ actually sitting in each hole [24, 25]. Indeed, according to Ref. [24], the free energy $F$ of the flux line interacting with a hole is essentially modified with increasing $n_{\mathrm{v}}$ (Fig. 13). It is well known that an
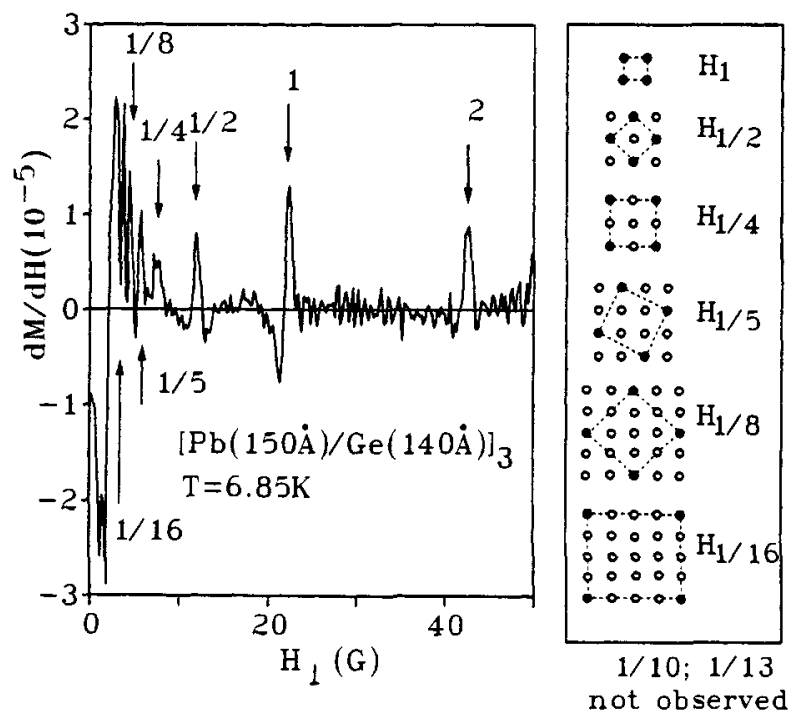

Fig. 12. The derivative $\mathrm{d} M / \mathrm{d} H$ for a $[\mathrm{Pb}(150 \AA) / \mathrm{Ge}(140 \AA)]_{3}$ multilayer with a square lattice of holes $(r=0.1 \mu \mathrm{m})$ at $6.85 \mathrm{~K}$ is shown on the left side. The matching fields are indicated by the arrows. The corresponding flux patterns, for the fractional quantization, are shown for one unit cell (see on the right side). The filled holes represent the holes occupied by flux lines. 


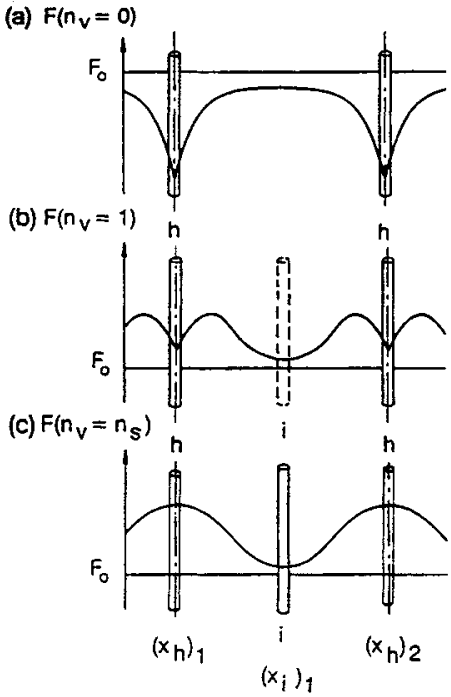

Fig. 13. The free energy $F(x)$ of a flux line interacting with a hole is shown as a function of the number of flux lines $n_{\mathrm{V}}$ : (a) $n_{\mathrm{V}}=0$, (b) $n_{\mathrm{V}}=1$ and (c) $n_{\mathrm{v}}=n_{\mathrm{s}}$, with $n_{\mathrm{s}}$ a certain saturation value (after Ref. [24]). $F_{0}$ is the free energy due to the self energy of the vortex and of the hole with magnetic flux quanta [24]. The symbol $h$ denotes the hole and $i$ the interstiatial position.

empty hole $\left(n_{\mathrm{v}}=0\right)$ always attracts a vortex since $F(x)$ is monotonically decreasing at $x \rightarrow x_{\mathrm{h}}$ [Fig. 13(a)]. The free energy $F(x)$ changes, however, when $n_{\mathrm{V}} \geqslant 1$ [Fig. 13(b)]. In this case a potential barrier appears between the dips at holes $\left(x=x_{h}\right)$ and shallow minima at interstitial positions $\left(x=x_{i}\right)$. When the number of flux lines reach a certain saturation number $n_{\mathrm{S}}[24]$ given by:

$n_{\mathrm{S}} \sim \frac{\kappa r}{2 \lambda(T)}$

a hole can not capture the next $\left(n_{\mathrm{S}}+1\right)$ th flux line since the dip at $x=x_{h}$ disappeared and the flux lines are repelled by a hole [Fig. 13(c)]. In eq. (10) $\kappa$ is the Ginzburg-Landau parameter.

The variation of free energy $F(x)$ determines the pinning force $f_{p} \sim \partial F(x) / \partial x$. This force depends on the number $n_{\mathrm{V}}$ of flux lines in the hole in the following way [24]:

$f_{p}=\frac{2 \pi}{\kappa}\left(1-\frac{2 n_{v} \lambda}{\kappa r}\right)$

We clearly see that the maximum pinning force $(2 \pi / \kappa)$ is provided by an empty hole as indicated by the steep slopes in $F(x)$ at $\left(x_{\mathrm{h}}\right)_{1},\left(x_{\mathrm{h}}\right)_{2},\left(x_{\mathrm{h}}\right)_{3}$ [see Fig. 13(a)]. Starting from a certain saturation number $n_{\mathrm{S}}$, the holes repel the $\left(n_{\mathrm{S}}+1\right)$ th vortex and $f_{p}=0$ at $x_{\mathrm{h}}$ while $f_{p}<0$ around $x_{\mathrm{h}}$. The vortices in this case are loosely bound at the interstitial positions [Fig. 13(c)].

A very interesting situation occurs however in the intermediate case $1 \leqslant n_{\mathrm{V}}<n_{\mathrm{S}}$ [Fig. 13(b)]. Energetically, it is better to have quasibound vortices at interstitial sites, since $F\left(x_{\mathrm{i}}\right)<F\left(x_{\mathrm{h}}\right)$. But if, due to a thermal activation, a vortex comes to the position $x=x_{\mathrm{h}}$ it will be trapped, since the pinning force $f_{p} \sim \partial F / \partial x$ at $x=x_{\mathrm{h}}$ is larger $\left(f_{p}\left(x_{\mathrm{h}}\right)>f_{p}\left(x_{\mathrm{i}}\right)\right)$. In this situation we have a lower energy at $x=x_{i}$, whereas the strongest pinning occurs at $x=x_{\mathrm{h}}$. If the vortices are indeed trapped by the holes for $1 \leqslant n_{\mathrm{V}}<n_{\mathrm{S}}$, the pinning occurs at sites not corresponding to the lowest energy but to the largest possible pinning force. This may be an interesting example of a non-equilibrium pinning phenomenon.

For $n_{v}>n_{\mathrm{S}}$ the occupation of the interstitial positions, with a much weaker pinning, becomes possible [25]. The existence of quasibound vortices at interstitial positions has been confirmed by measuring the flux creep rate and will be published elsewhere [26].

The absence of the rational peaks in fields $H>H_{1}$ (see Fig. 12) is probably due to the formation of the quasibound vortices at the interstitial positions [Fig. 13(b)]. Instead of producing a "rational" array consisting of two and one flux line per hole, the flux lines repelled by the holes seem to occupy the interstices, thus keeping unchanged the $n_{\mathrm{V}}=1$ occupancy. At the second matching field $H_{2}$ all positions $x_{i}$ are occupied and energetically it is more favourable to have at $\mathrm{H}>\mathrm{H}_{2}$ two flux lines per hole, instead of putting two flux lines around the same interstitial position $x_{\mathrm{i}}$ [27]. This may only occur at temperatures sufficiently high to activate vortices through the barrier between $x_{i}$ and $x_{h}$ [see Fig. 10 and 13(b)].

The presence of integer matching peaks can be explained if we assume the formation of multi-quanta vortices at the holes [27]. In this model two-quanta vortices can be formed if $r>r_{\mathrm{c}}$, where $r_{\mathrm{c}}$ is given by

$r_{\mathrm{c}}=\left(\xi a_{v}^{2}\right)^{1 / 3}$

with $a_{\mathrm{v}}$ being the vortex lattice period. For our $\mathrm{Pb} / \mathrm{Ge}$ multilayers $\lambda(0)$ and $\xi(0)$ can be evaluated using the dirty limit expressions $\xi(0)=\left(\xi_{0} l\right)^{1 / 2}$ and $\lambda(0)=\lambda_{0}\left(\xi_{0} / l\right)^{1 / 2}$, where $\xi_{0}=83 \mathrm{~nm}$ and $\lambda_{0}=37 \mathrm{~nm}$ are the clean limit coherence length and penetration depth of $\mathrm{Pb}$ [1]. From the measured upper critical field $H_{c 2}(0)$ we find that $\xi(0) \cong 12 \mathrm{~nm}$ and therefore $l \cong 17 \AA, \lambda(0)=260 \mathrm{~nm}$ and $\kappa \cong 21$. Inserting the "attempted" vortex lattice period at $H=H_{2} a_{\mathrm{v}}=0.5 \mu \mathrm{m}$ in eq. (12), we find that $r_{\mathrm{c}} \cong 0.14 \mu \mathrm{m}$, i.e. the radius of the holes $r \cong 0.1 \mu \mathrm{m}$ is indeed close to the calculated $r_{c}$ value. We note here, that eq. (12) is only a rough approximation, since it has been derived for bulk three dimensional superconductors, and it should be modified for layered structures. It is well known (see, for example Ref. [1]) that in thin films the repulsion between vortices is stronger, so we may expect a smaller $r_{c}$ value for superconducting multilayers. Therefore, the radius of the holes in our case seems to be sufficiently large to provide the formation of multiple-quanta vortices at integer matching fields. The highest possible integer matching peak $n_{\mathrm{S}}=4$ can be calculated from eq. (10) by inserting $\kappa \cong 21, r \cong 0.1 \mu \mathrm{m}, \lambda \cong 0.26 \mu \mathrm{m}$. This is in good agreement with the largest number of integer peaks $H / H_{1}=3 \cong n_{\mathrm{S}}$ observed. Increasing the diameter of holes leads to the growth of the saturation number $n_{\mathrm{s}}$ and as a result more matching peaks are observed, which is in agreement with eq. (10) (compare curves for $r \approx 0.1 \mu \mathrm{m}$ and $r \approx 0.2 \mu \mathrm{m}$ in Fig. 14).

The decrease of the integer matching peaks at lower temperatures [Fig. 10(b), (c) [ can be explained as a result of freezing out of the thermal activation which is necessary to overcome the energy barrier between different configurations of vortices [see Fig. 13(b)]. It is important to note here that this critical value $n_{\mathbf{S}}$ is determined by the ratio $r / \lambda(T)[25]$ and therefore grows as temperature goes down.

The experimentally found rational peaks in the first period (Fig. 12) at $H / H_{1}=1 / 16 ; 1 / 8(1 / 9 ?) ; 1 / 5 ; 1 / 4 ; 1 / 2$ are 


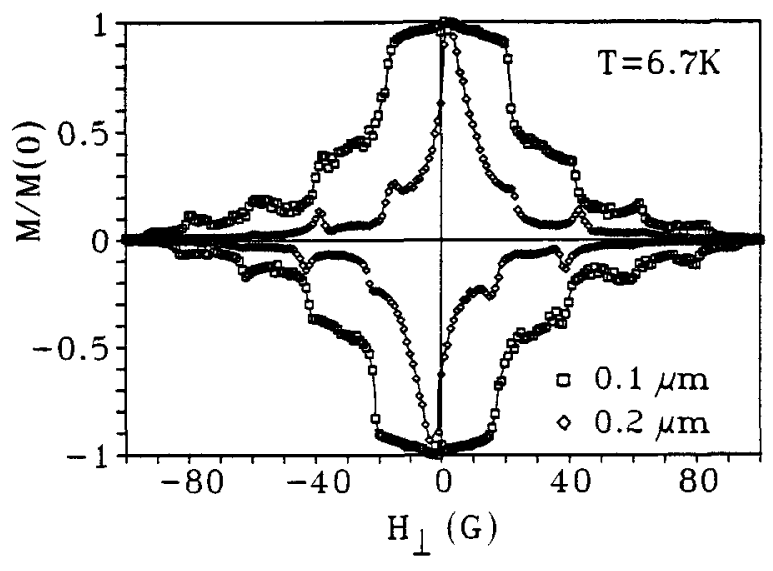

Fig. 14. Comparison of the $[\mathrm{Pb}(150 \AA) / \mathrm{Ge}(140 \AA)]_{3}$ multilayers with a lattice of holes with different radii $r=0.1 \mu \mathrm{m}$ and $r=0.2 \mu \mathrm{m}$.

very different from those observed for Josephson networks $(1 / 4 ; 1 / 3 ; 2 / 5 ; 1 / 2 ; 3 / 5 ; 2 / 3 ; 3 / 4)[16-21]$. For $H<H_{1}$, the problem of the interaction between $n$ flux lines, which are repelling each other, in the presence of $N$ pinning centers $(N>n)$ is analogous to the interaction between the $n$ negatively charged particles in the presence of $N$ available acceptor sites $(N>n)$ in compensated weakly doped semiconductors (see for example the description of the Coulomb gap in the impurity band in doped semiconductors [28]). The optimization of the flux line positions for $H<H_{1}$ leads to the formation of larger square flux lattices with the period $d_{k / l}$ with sites exactly falling onto the sites of the square lattice of holes with the period $d$. This constraint immediately gives $d_{k / l}=\sqrt{l^{2} d^{2}+k^{2} d^{2}}=d \sqrt{l^{2}+k^{2}}$. Here $l$ and $k$ are integer numbers. Therefore, we should expect the fractional peaks at

$H=H_{m}=\frac{\phi_{0}}{d_{k / l}^{2}}=\frac{H_{1}}{l^{2}+k^{2}}$

The peaks, calculated for different $k$ and $l$ (in the order of increasing $l^{2}+k^{2}$ )

$H_{1 / 1}=\frac{1}{2} ; \quad H_{0 / 2}=\frac{1}{4} ; \quad H_{1 / 2}=\frac{1}{5} ;$

$H_{2 / 2}=\frac{1}{8}\left(H_{0 / 3}=\frac{1}{9} ?\right) ; \quad H_{0 / 4}=\frac{1}{16}$

are in good agreement with our experimental data. It is worth emphasizing here the essential difference between these peaks and the peaks at $H / H_{1}=\frac{1}{4}, \frac{1}{3}, \frac{2}{5}, \frac{1}{2}, \frac{2}{3}, \frac{3}{4}$ found for a square network [16]. Five out of these seven peaks are definitely not seen in the flux patterns in the presence of the submicrometer holes (see Fig. 12, where on the right side the corresponding flux configurations are shown). The naive expectation of the identity between rational matching peaks in a network and a lattice of small holes turns out to be incorrect.

A crucial role for the observation of the rational matching peaks is played by a stronger interaction $U_{\mathrm{vv}}$ between the flux lines in the multilayers where most probably the interaction potential decays only as $1 / r$, like in $2 \mathrm{D}$ systems, whereas in $3 \mathrm{D}$ superconductors the interaction is much weaker $U_{\mathrm{vv}} \propto \exp (-r / \lambda)[1]$.

Concluding this section, we would like to point out that we clearly showed that unusual flux line matching effects can be observed in $\mathrm{Pb} / \mathrm{Ge}$ multilayers due to the flux lines confinement by a square lattice of submicrometer holes.
Sharp peaks in the $M\left(H_{\perp}\right)$ and $j_{c}\left(H_{\perp}\right)$ curves at integer and rational matching fields are found. The flux line patterns, corresponding to these peaks, have been identified. The existence of multi-quanta vortices trapped into the holes is confirmed and the possibility of a strong enhancement of $j_{c}$ by a lattice of well defined is demonstrated, i.e. "quantum design" of $j_{c}$ is possible.

\section{Conclusion}

By optimizing the confinement potential for the superconducting condensate and for the flux lines, a substantial enhancement of the superconducting critical parameters $\left(H_{c}\right.$ and $j_{c}$ ) can be achieved in mesoscopic superconducting systems and nanostructural superconductors. This enhancement is closely related to the confinement and quantization effects and it clearly demonstrates that the "quantum design" of the superconducting critical parameters is possible.

\section{Acknowledgements}

This research has been supported by the Belgian Inter-University Institute for Nuclear Sciences (IIKW), the Inter-University Attraction Poles (IUAP), the Concerted Action (GOA) programs, the Belgian High Temperature Superconductivity Incentive and Concerted Action Programs. L. Gielen and M. Baert are Research Assistants of the Belgian Institute for the Encouragement of Scientific Research in Industry and Agriculture (I.W.O.N.L.), V. V. Metlushko is a Postdoctoral Fellow supported by the Belgian Science Policy Office, while C.V.H. is Senior Research Associate of the Belgian National Fund for Scientific Research (N.F.W.O.).

\section{References}

1. de Gennes, P. G., "Superconductivity in Metals and Alloys" (AddisonWesley, New York 1989).

2. Abrikosov, A. A., "Fundamentals of the Theory of Metals" (NorthHolland, Amsterdam 1988).

3. Saint-James, D., Phys. Lett. 15, 13 (1965).

4. Vloeberghs, H., Moshchalkov, V. V., Dhallé, M., Neuttiens, G., Van Haesendonck, C. and Bruynseraede, Y., Phys. Rev. Lett. 69, 1268 (1992).

5. Tinkham, M., "Introduction to Superconductivity" (McGraw-Hill, New York 1975).

6 Little, W. A. and Parks, R. D., Phys. Rev. Lett. 9, 9-13 (1962).

7. Fink, H., López, A. and Maynard, R., Phys. Rev. B26, 5237 (1985).

8. Moshchalkov, V. V., Gielen, L., Dhallé, M., Van Haesendonck, C. and Bruynseraede, Y., Nature 361, 617 (1993).

9. Huebener, R. P., "Magnetic Flux Structures in Superconductors" (Springer-Verlag, Berlin, Heidelberg, New York 1979).

10. Larkin, A. I. and Ovchinnikov, Yu. N., J. Low Temp. Phys. 34, 409 (1979).

11. Civale, L. et al., Phys. Rev. Lett. 67, 648 (1991).

12. Gerhäuser, W. et al., Phys. Rev. Lett. 68, 879 (1992).

13. Daldini, O., Martinoli, P., Olsen, J. L. and Berner, G., Phys. Rev. Lett. 32, 218 (1974).

14. Hebard, A. F., Fiory, A. T. and Somekh, S., IEEE Transactions on Magnetics 1, 589 (1977); A. T. Fiory, A. F. Hebard and S. Somekh, Appl. Phys. Lett. 32 (1), 73 (1978).

15. Lykov, A. N., Solid State Commun. 86, 531 (1993).

16. Pannetier, B., Ghaussy, J., Rammal, R. and Villegier, J. C., Phys. Rev. Lett. 53, 1845 (1984).

17. Straley, J. P. and Barnett, G. M., Phys. Rev. B48, 3309 (1993).

18. Lobb, J., Physica B152, 1 (1988).

19. Halsey, Thomas C., Phys. Rev. B 31, 5728 (1985).

20. Villain, J., J. Phys. C10, 1717 (1977).

21. Hallen, H. D. et al., Phys. Rev. Lett. 71, 3007 (1993).

22. Clem, J. R., J. Low Temp. Phys. 18, 427 (1975). 
23. Bruynseraede, Y. et al., Physica Scripta T42, 37 (1992).

24. Mkrtchyan, G. S. and Shmidt, V. V., Sov. Phys. JETP 34, 195 (1972).

25. Khalfin, I. B. and Shapiro, B. Ya., Physica C207, 359 (1993).

26. Baert, M., Metlushko, V. V., Jonckheere, R., Moshchalkov, V. V. and Bruynseraede, Y., submitted to Europhys. Lett.
27. Buzdin, A. I., Phys. Rev. B47, 11416 (1993).

28. Shklovskii, B. I. and Efros, A. L., in "Electronic Properties of Doped Semiconductors" (Edited by M. Cardona, P. Fulde and H.-J. Queisser), Springer Series in Solid-State Sciences (Springer, Berlin 1984), and references therein. 